\title{
Incidental finding on X-ray shoulder in young male patient
}

\begin{abstract}
Esophageal leiomyoma is a benign smooth-muscle tumor of the esophagus. It is the commonest benign tumor of the esophagus. It is most frequently presents in young and middle age groups from 20-50 years of age. The overall incidence is around 8-43 per 10,000 autopsy series. ${ }^{1}$ Clinical presentation usually depends on the size of tumor. They mainly involve the mid-to-distal esophagus. ${ }^{1}$ Like other leiomyomas, they comprise of smooth muscle overgrowth. I discussed here a patient coming for his shoulder swelling and incidentally diagnosed superior paraspinal mass incidentally and further work-up was done and diagnosed esophageal leiomyoma histopathologically.
\end{abstract}

Keywords: esophagus, abnormalities, CT, MR, tumor, location, symptoms, esophageal obstruction, bleeding, endoscopy
Volume 9 Issue 2 - 2018

\section{Sushila B Ladumor}

Department of Clinical Imaging, Hamad Medical Corporation, Assistant Professor, Clinical Radiology, Weil Cornel Medical College, Qatar

Correspondence: Sushila B Ladumor, Consultant Radiologist, Clinical Imaging Department, Hamad Medical Corporation, Assistant Professor in Clinical Radiology, Weil Cornel Medical College, Doha, Qatar (WCMC-Q),

Email drsbladumor@yahoo.com

Received: September 29, 2017 | Published: March 22, 2018

\section{Introduction with background}

Benign tumors of the esophagus are rare, accounting for less than $10 \%$ of esophageal tumor. ${ }^{2,3}$ In benign tumors, leiomyomas represent about two-third. ${ }^{4}$ More than $90 \%$ of esophageal tumors are malignant. Esophageal leiomyoma is the most common benign esophageal tumor, the rest of benign tumors being extremely rare. It occurs more frequently in men, male female ratio is $2: 1$. Symptoms are nonspecific, common causes are dysphagia and epigastric pain, but $50 \%$ of patients may be asymptomatic. We present a patient with esophageal leiomyoma discovered incidentally during investigations for right shoulder swelling.

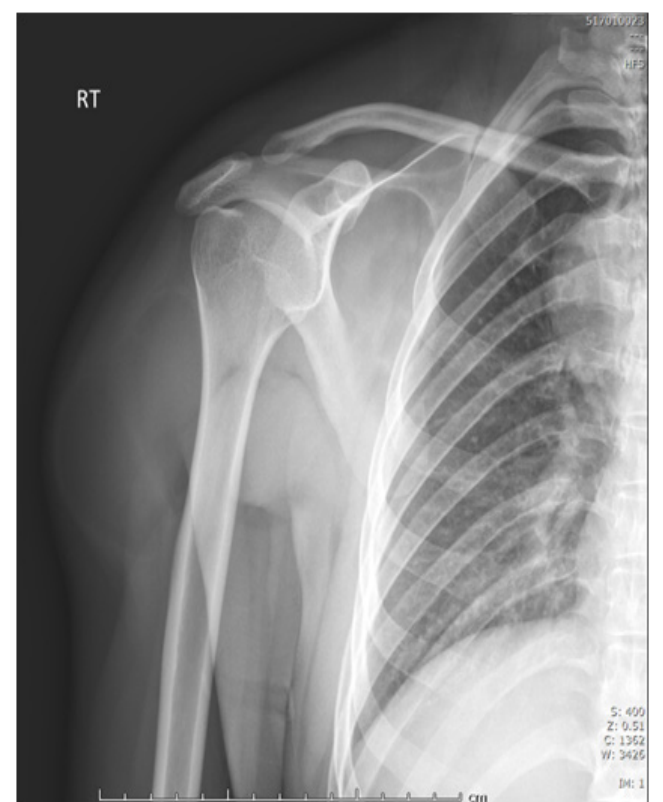

Figure Frontal X-ray Shoulder: (No previous exam of shoulder or X-ray chest available.) There is soft tissue swelling on lateral aspect of upper right humerus which shows similar appearance as subcutaneous fat, likely representing lipoma. Incidental findings:Well defined soft tissue lesion at upper mediastinum on right side below medial end of right clavicle. Medial margin of mass is not appreciated.

\section{Case report}

\section{Clinical history}

33 years old male without any past medical history: He noticed right shoulder swelling before 20 days of presentation. There was no pain, no decrease range of motion, no other swelling in the body and no previous swellings like that before.

Figure $1 \& 2$.

\section{Clinical presentation: depends on the size of tumor}

Figure 3-5

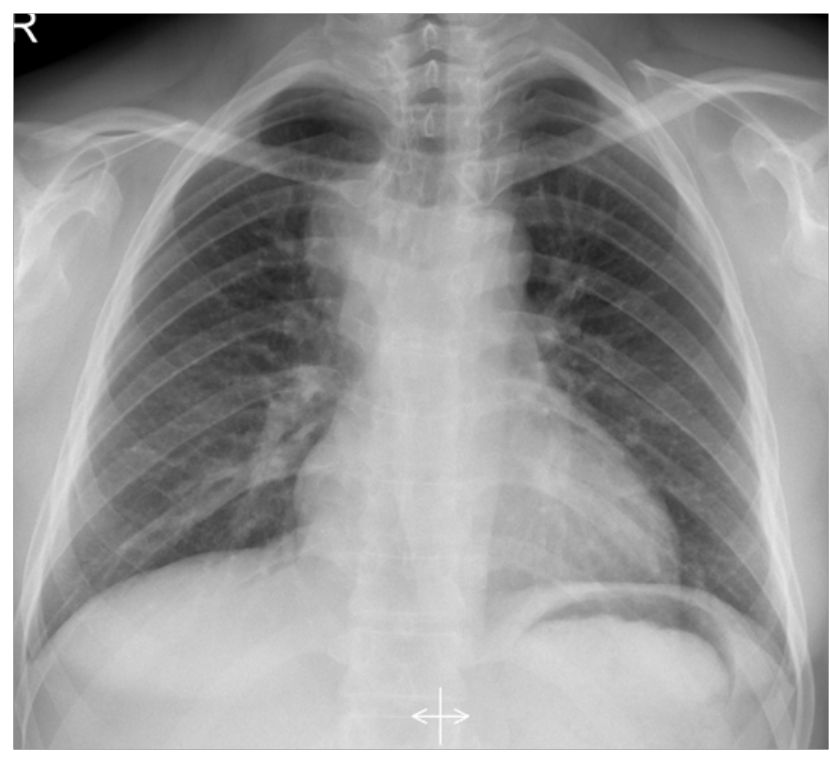

Figure 2 X-Ray Chest frontal projection: Re-demonstration of right sided superior mediastinal mass at supra hilar region with well-defined lateral border. Non visualization of medial border and form the obtuse angle with adjacent lung. Lung fields are clear. No pleural effusion. Heart size appears within normal limit. The mass shows widening of right parasternal stripes representing posterior mediastinal origin. No calcification, no gross bony abnormality seen. 


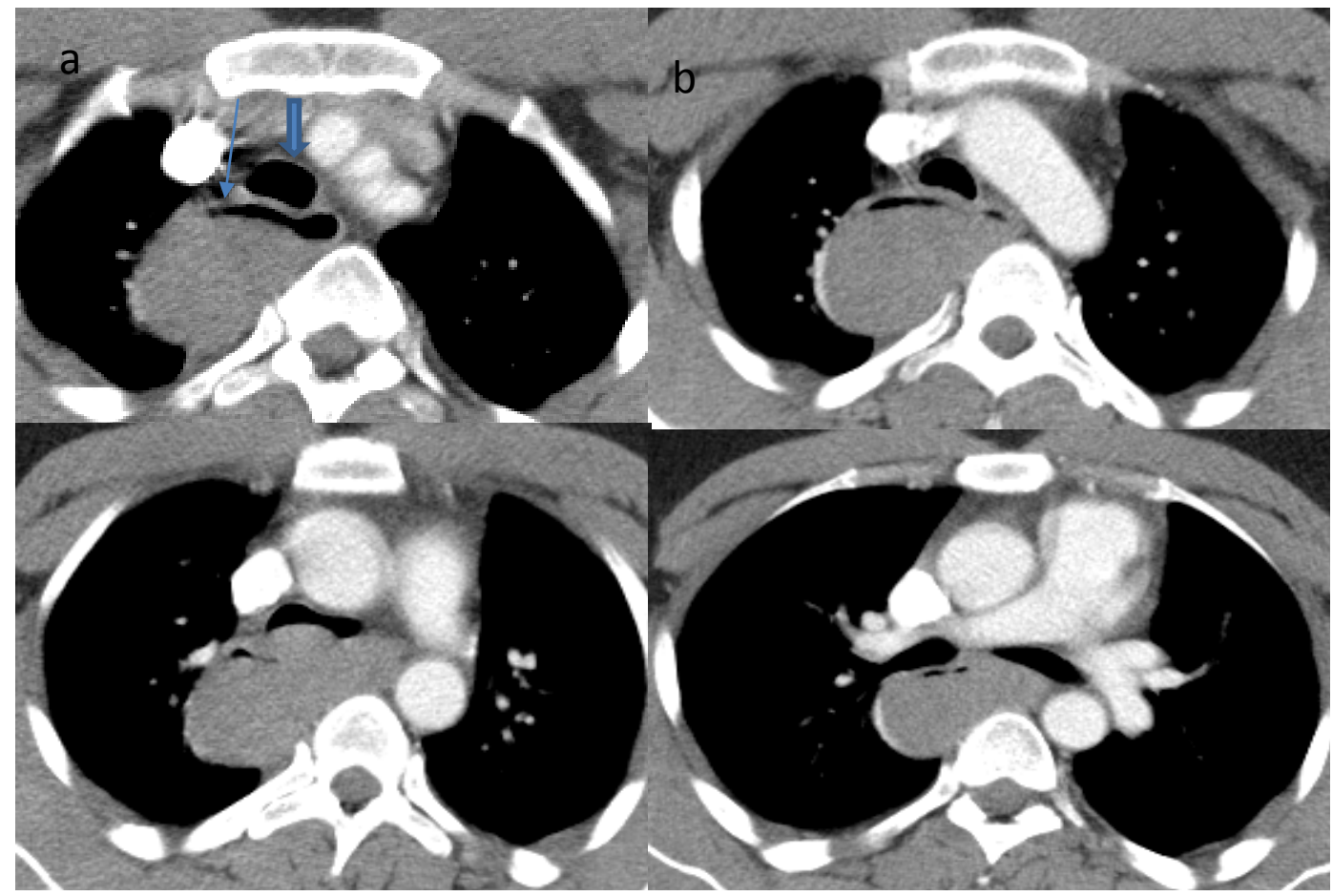

Figure 3(A-D) Contrast enhanced CT CHEST Axial Images: Soft tissue mass in right posterior superior mediastinum with mild enhancement. It appears inseparable to upper esophagus with air filled stretched esophagus (Thin arrow in image \# a) which extend below level of carina and mild impression over posterior aspect of trachea (Thick arrow in image \#b). No calcification. No connection with neural foramina. No bone destruction.

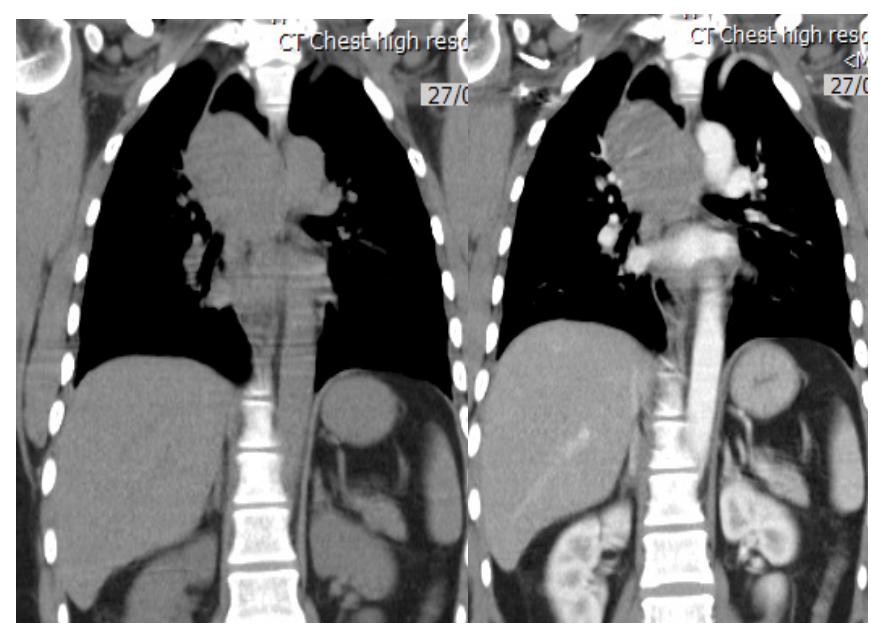

Figure $4 \mathrm{CT}$ Coronal reformation. Pre-contrast (A) and post-contrast (B): Mildly enhancing superior mediastinal mass appears inseparable from esophagus with proximal esophageal dilatation.

\section{Endoscopic ultrasound from system}

Endoscopic US (Linear EUS scope used): Report: A large 4 x $6 \mathrm{~cm}$ hypoechoic mass was noted in mediastinum. The layer of origin of the mass could not be appreciated due to its large size. On puncturing the lesion was soft in consistency.

\section{Findings}

Figure $1 \mathrm{X}$-ray shoulder frontal projection: done first demonstrates:
(No previous exam of shoulder or X-ray chest available.) There is soft tissue swelling on lateral aspect of upper right humerus which shows similar appearance as subcutaneous fat, likely representing lipoma.

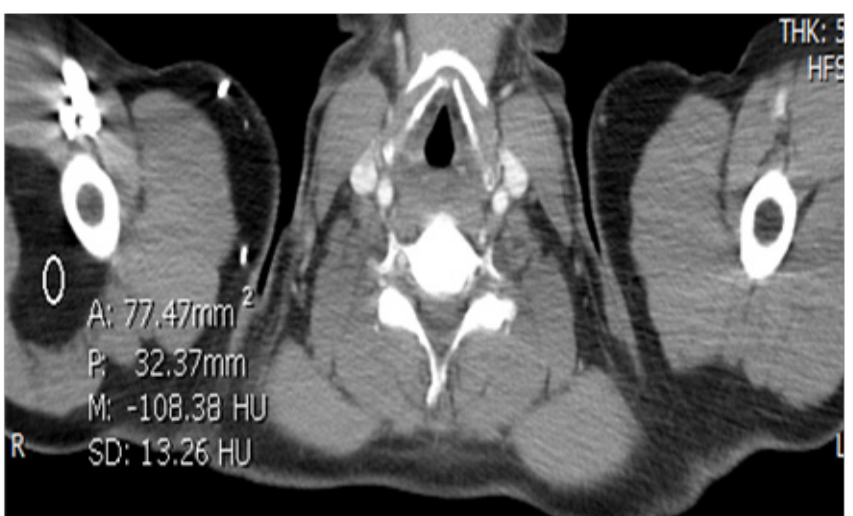

Figure 5 Post-contrast CT chest upper cut axial Image: Demonstrates fat containing mass adjacent to right proximal humerus representing lipoma. No appreciable enhancement. No definite underlying bone destruction.

Incidental findings: Well defined soft tissue lesion at upper mediastinum on right side below medial end of right clavicle. Medial margin of mass is not appreciated.

Figure 2 X--ray chest was recommended: X-Ray chest frontal projection: Demonstrates: Re-demonstration of right sided superior mediastinal mass at supra hilar region with well-defined lateral border. Non visualization of medial border and form the obtuse angle with adjacent lung. Lung fields are clear. No pleural effusion. Heart size appears within normal limit. The mass shows widening of right 
parasternal stripes representing posterior mediastinal origin. No calcification, no gross bony abnormality seen. This includes wide differential diagnosis of posterior mediastinal mass: Neurogenic tumor, Non-neurogenic tumor, Infection, Inflammation, Vascular, lymphadenopathy, extramedullary hematopoiesis, foregut duplication cysts and Thoracic meningocoele. CT scan of Chest with Intravenous Contrast advised.

Figure 3-5 CT Chest Without \& with Intravenous Contrast: Findings: Soft tissue mass in right posterior superior mediastinum with mild enhancement. It appears inseparable to upper esophagus with air filled stretched esophagus which extend below level of carina and mild impression over posterior aspect of trachea. No calcification. No connection with neural foramina. No bone destruction. Also demonstrates fat containing mass adjacent to right proximal humerus representing lipoma. No appreciable enhancement. No definite underlying bone destruction. Imaging findings are most likely representing esophageal lesion with high possibility of esophageal leiomyoma.

Endoscopy with Biopsy recommended for confirmation:

Endoscopic Us: A large 4 x $6 \mathrm{~cm}$ hypoechoic mass was noted in mediastinum. The layer of origin of the mass could not be appreciated due to its large size. On puncturing the lesion was soft in consistency.

\section{Biopsy taken}

\section{Histopathology: Leiomyoma}

Symptoms: Small tumors $(<5 \mathrm{~cm})$ : usually no symptoms

Large tumors: may cause dysphagia, regurgitation, esophageal obstruction, chest pain, cough, or bleeding (rare)

\section{Radiographic features}

The usual imaging findings of esophageal leiomyoma describe it as a smoothly marginated, round or lobulated mass projecting to one or both sides of the mediastinum along the course of the esophagus.

\section{A. Fluoroscopy}

On barium swallow, may be seen as a discrete ovoid mass that is well outlined by barium. It's usually forms slightly obtuse angles with the esophageal wall.

\section{B. CT Findings include}

I. Ovoid intramural solitary homogeneous mass with a smooth surface or esophageal wall thickening with the presence of calcifications is almost pathognomonic.

II. Narrowing of the esophageal lumen and may displace the esophagus

III. Moderate diffuse contrast-enhancement

IV. No signs of invasion of adjacent tissue

\section{MRI Findings include}

I. Ovoid intramural solitary mass with a smooth surface with signal intensity typical of smooth muscle in $\mathrm{T} 1$ and $\mathrm{T} 2$ weighted sequences, No fatty component. (Calcification better seen by CT than MRI)
II. Narrowing of the esophageal lumen and may displace the esophagus

III. Moderate diffuse contrast-enhancement

IV. No signs of invasion of adjacent tissue

\section{Differential diagnosis}

Imaging differential considerations of esophageal mass include:

\section{Esophageal GIST}

II. Esophageal carcinoma

III. Esophageal leiomyosarcoma

IV. Esophageal hamartoma

V. Other mediastinal tumors arising close to the esophagus

\section{Foreign body}

\section{Discussion}

If leiomyoma growth is intraluminal, they encroach on the mucosa and appear as sessile or pedunculated polyps. Ulceration of the overlying mucosa is rare, in contrast to gastric leiomyomas as it is common in gastric leiomyoma. Esophageal leiomyomas can encircle the whole esophagus in its lower third.

Esophageal leiomyomas are usually solitary, ranging from 2 to $6 \mathrm{~cm}$ in diameter although tumors of several millimeters to $22 \mathrm{~cm}$ in diameter have also been reported. ${ }^{5}$ Particularly when large, they may be recognized as posterior mediastinal masses with mass effect on an adjacent organ. Multiple esophageal leiomyomas are rare, although have been reported in a patient with Alport syndrome. ${ }^{4}$ Esophagography reveals them, in profile, as smoothly round or crescentic filling defects. When viewed en face, they appear as round or lobulated filling defects, sharply outlined by the barium flowing around each side.

CT findings, esophageal leiomyomas are smoothly marginated, round or ovoid masses of muscle attenuation, located intramurally or eccentrically within the esophageal wall. Surrounding mediastinal fat is not usually disrupted. ${ }^{1}$

There have been few published accounts of the MR findings of esophageal leiomyomas. On T2-wighted images their signal is usually isointense, while in esophageal cancer a high-intensity signal is normally observed.

Leiomyoma may occur in all parts of the esophagus, but approximately $60 \%$ of leiomyomas occur in the lower third of the esophagus, $30-33 \%$ in the middle third and $7-10 \%$ in the upper third. ${ }^{1,5-7}$ Their relative rarity in the upper esophagus reflects the smaller amount of smooth muscle in that region.

Approximately 50 percent of patients usually have no symptoms, while some having degrees of dysphagia and substernal pain are the most common presenting symptoms. A tumor's size and growth characteristics are main factors in appearance of symptoms. Esophageal leiomyoma is slowly growing lesion and usually produce intermittent, gradual progressive symptoms. Occasionally, they encircle the esophagus in a serpentine or U-shaped fashion and cause obstructive symptoms. 


\section{Treatment and prognosis}

The overall prognosis of these benign tumors is excellent. If the tumor is small $(<5 \mathrm{~cm})$ and asymptomatic treatment is not necessary. Otherwise, surgical enucleation is recommended.,.$^{3,7}$

\section{Note}

Gastro-Intestinal surgeon consulted, explained his disease and treatment option is surgery but patient refused for surgery as he said I have no complaint and travelled to his original country.

\section{Conclusion}

Esophageal leiomyomas, located mainly in the middle or distal esophagus, are seen mainly eccentrically elevated filling defects at CT, lesions showing homogeneous low or iso attenuation of the mass. Esophagography, in which the tumor is seen as an intramural, eccentric, smoothly elevated, sessile lesion, varying in size, is the most reliable form of diagnosis.

\section{Acknowledgements}

None.

\section{Conflict of interest}

Authors declare there is no conflict of interest in publishing the article.

\section{References}

1. Yang PS, Lee KS, Lee SJ, et al. Esophageal leiomyoma: radiologic findings in 12 patients. Korean J Radiol. 2002;2(3):132-7.

2. Rabushka LS, Fishman EK, Kulman JE, et al. Diffuse esophageal leiomyomatosis in a patient with Alport syndrome. Radiology. 1991;179(1):176-8.

3. Ajay Punpale, Ashvin Rangole, Navin Bhambhani, et al. Leiomyoma of Esophagus. Ann Thorac Cardiovasc Surg. 2007;30(2):78-81.

4. Seremetis MG, de Guzman VC, Lyons WS, et al. Leiomyoma of the esophagus: a report of 19 surgical cases. Ann Thorac Surg. 1973;16:308316.

5. Xuefei Sun, Jiabang Wang, Guotao Yang. Surgical treatment of esophageal leiomyoma larger than $5 \mathrm{~cm}$ in diameter: A case report and review of the literature, J Thorac Dis. 2012;4(3):323-326.

6. Aurea P, Grazia M, Petrella F, et al. Giant leiomyoma of the esophagus. Eur J Cardiothorac Surg. 2002;22:1008-1010.

7. Peters JH, DeMeester TR. Esophagus and diaphragmatic hernia. In: Brunicardi FC, Andersen KD, Billiar RT, Dunn LD, Hunter GC, Pollock RE, editors. Schwartz's Principles of Surgery. 8th ed. New York: McGrawHill, 2005; 906. 